\title{
THE APPLICATION OF OZONE TO REDUCE THE COLORING INTENSITY OF AQUEOUS SOLUTIONS OF DYES USED IN THE TEXTILE INDUSTRY
}

\author{
Sergey Belov ${ }^{1 *}$, Grigoriy Naumchik ${ }^{1}$ \\ ${ }^{1} \mathrm{UO}$ BrSTU, Brest, Republic of Belarus
}

\begin{abstract}
The article discusses the mechanisms of interaction of ozone with aqueous solutions of organic dyes. Two different dyes with different types of chromophore systemwere investigated: azo dye and thiazine dye. A detailed methodology for performing experimental studies makes it possible to accurately dose ozone into the reaction mixture. The data of experimental studies are presented indicating the effectiveness of the use of ozone for the destruction of dyes in aqueous solutions. The specific doses of ozone were established to reduce the color intensity of the studied dye solutions to standard values.
\end{abstract}

Keywords: Ozone, dyes, destruction, color intensity, wastewater, textile industry.

\section{Introduction}

Wastewater from textile factories is characterized by a high content of pollutants. They contain up to $1000 \mathrm{mg} / \mathrm{L}$ of suspended solids and are characterized by high chemical oxygen demand (COD) which is determined by the content of organic pollutants in colloidal and soluble forms [1, 2, 3]. However, one of the most characteristic indicators of wastewater pollution from textile enterprises is their color intensity by the degree of dilution $[1,4,5]$.

The color of waste water in the production of textiles is mainly due to the widespread use of organic dyes. Depending on the specialization of the enterprise, fibers, fabrics and finished products are dyed during the production process. A significant part of the dyes (from $10 \%$ to $50 \%$ ) during the dyeing process remains in the waste technological solutions and in the wash water formed after washing the dyed items [1, 6, 7]. As a result, the intensity of color in terms of the degree of dilution in the wastewater of textile enterprises using dyes in the production cycle can reach 1: 1000. At the same time, the intensity of coloring in terms of the degree of dilution in domestic and industrial waste water

\footnotetext{
*Corresponding author: siarhei.bialou@gmail.com
} 
discharged both into the municipal sewer network and into natural water bodies should not exceed 1:20 [1,4].

Synthetic dyes currently used for dyeing textile materials are persistent organic compounds that are difficult to biooxidize $[1,4,8]$. Therefore, when entering municipal wastewater these compounds pass practically unchanged through biological treatment facilities and enter natural water bodies having a negative impact on their ecosystem [9]. The following methods can be used to remove dyes that cause the color of wastewater: reagent coagulation, electrocoagulation, sorption, barometric methods, destructive methods (oxidation, reduction, UV treatment) [1, 8]. Each method has its own advantages and disadvantages and should be applied based on the conditions of a specific production. However, one of the most versatile methods is ozonation [10,11, 12, 13]. Unlike reagent coagulation and electrocoagulation, ozonation does not form precipitates, unlike sorption,ozonation does not generate spent regeneration solutions compared to barometric methods so ozonation is more reliable and easy to operate $[14,15,16]$.

The main value of ozone for removing pollutants, in contrast to other reagents, lies in its high reactivity which makes it possible to destroy practically all water-soluble organic dyes using ozonation. In this case, ozone exhibits its high oxidizing properties at any $\mathrm{pH}$ value [17]. Ozone interacts most vigorously with unsaturated bonds [18]:

ethylene $>\mathrm{C}=\mathrm{C}>$, azomethine $>\mathrm{c}=\mathrm{N}-$, azo $-\mathrm{N}=\mathrm{N}-$, thiocarbonyl $>\mathrm{C}=\mathrm{S}$.
However, with a carbonyl bond $>\mathrm{c}=\mathrm{O}$ the reaction is not very active.

When developing a technology for purifying waste water from dyes, the reaction with an azo bond is of greatest interest, since most dyes used in light industry are azo dyes in terms of chromophore structure [19]. Literature sources indicate that the interaction of ozone with azo bonds occurs with the breaking of the double bond and the formation of intermediate addition products which are instantly hydrolyzed in an aqueous medium with the final breaking of the azo bond and the formation of oxy compounds [18]. This reaction mechanism, in principle, can be similar to the interaction of ozone with an ethylene bond [20]:

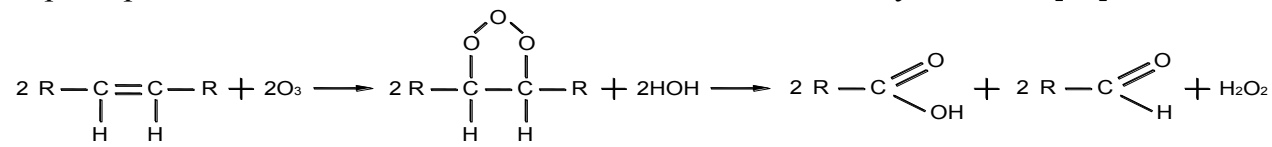

However, it was found when searching in the literature that the mechanism of the reaction of ozone with azo bonds of dyes in aqueous solutions has not been studied in detail.

The interaction of ozone with aromatic compounds has been studied in more detail which is also of great theoretical importance for determining the mechanism of interaction of ozone with dyes in aqueous solutions. All synthetic dyes contain aromatic residues in their molecules. Therefore, various derivatives of aromatic hydrocarbons are formedduring the decomposition of dyes. These compounds, as a rule, are not colored or weakly colored but they are resistant to biological oxidation and are highly pathogenic for aquatic ecosystems. The interaction of ozone with aromatic compounds also occurs through the mechanism of the addition of ozone to the double bond and subsequent hydrolysis of the resulting intermediate compound [6].

The relevance of the development of effective methods for the removal of dyes from the wastewater of the textile industry has recently increased in connection with the increasing requirements for the protection of the surrounding aquatic environment. [7, 21]

We investigated in this work the efficiency of using ozone to reduce the color intensity of model solutions prepared on the basis of two dyes with different chemical structures of the chromophore. 
We studied model solutions of the following dyes:

1) Acid metal complex azo dye "Lanasyn Black M-DL" produced by the Swiss company "Clariant". The dye was obtained at OJSC Brest Stocking Plant as one of the most widely used in production and difficult to remove in wastewater treatment.

2) Thiazine dye "Methylene blue". This dye, by its nature, is not an azo dye, it is highly resistant to oxidants since it itself has weak oxidizing properties. This dye has a relatively low molecular weight and a very high extinction coefficient $[22,23]$.

The chemical formulas of the above dyes are shown in Figure 1.

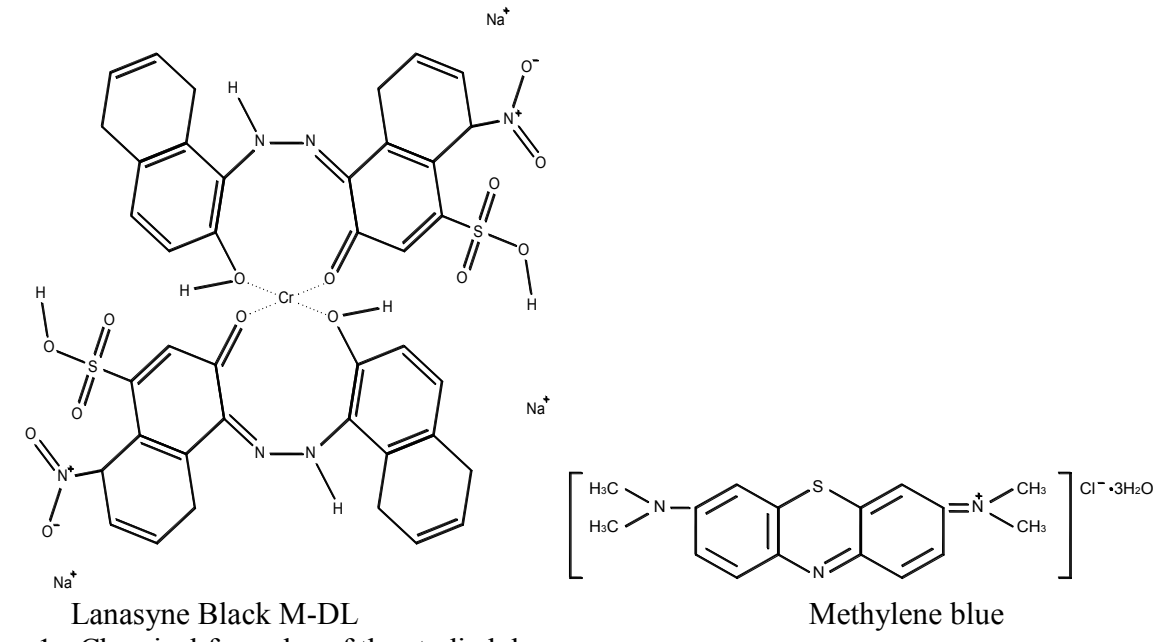

Figure 1 - Chemical formulas of the studied dyes

\section{Method}

We used solutions of dyes "Lanasyn Black M-DL" and "Methylene blue" in distilled wateras an object of research. The concentration of the initial solutions was $1 \mathrm{~g} / \mathrm{L}$. Working solutions of dyes "Lanasyn Black M-DL" with a concentration of $30 \mathrm{mg} / \mathrm{L}$ (color intensity at a dilution ratio of 1:200) and "Methylene blue" with a concentration of $4 \mathrm{mg} / \mathrm{L}$ (color intensity at a dilution rate of 1: 400)were prepared from these solutions by the dilution method.

The technique presented in the article [24] was usedto determine the intensity of the color by the degree of dilution.

The method in which gaseous ozone is introduced into the aqueous solution of the treated substance was not used since this method of introducing ozone does not allow to accurately determine the amount of ozone that has reacted. It is impossible when bubbling a solution to determine how much ozone has dissolved in an aqueous solution in the bubble column and reacted with substances dissolved in this solution and how much ozone remained in the gas phase and got into the ozone destructor.The dose of ozone when it is introduced into the treated aqueous solution by bubbling is a value that depends on the average diameter of the bubbles coming out of the gaseous ozone disperser, the temperature of the treated aqueous solution which affects the ozone solubility, the contact time of the solution with ozone bubbles which depends on the height of the column of the treated solution, concentration of ozone in the supplied gas mixture, duration of the ozonation process and other factors. Ozone was introduced by the method of its precise dosage in the form of an aqueous solutionto exclude the influence of the above factors on the efficiency 
of ozonation. An aqueous solution of ozone was obtained in a glass column filled with distilled water by bubbling water with an ozone-oxygen mixture. Distilled water was bubbled through a porous dispersant. In this way, an aqueous solution of ozone dissolved in distilled water was obtained. The scheme of the installation for the dissolution of ozone in water is shown in Figure 2.

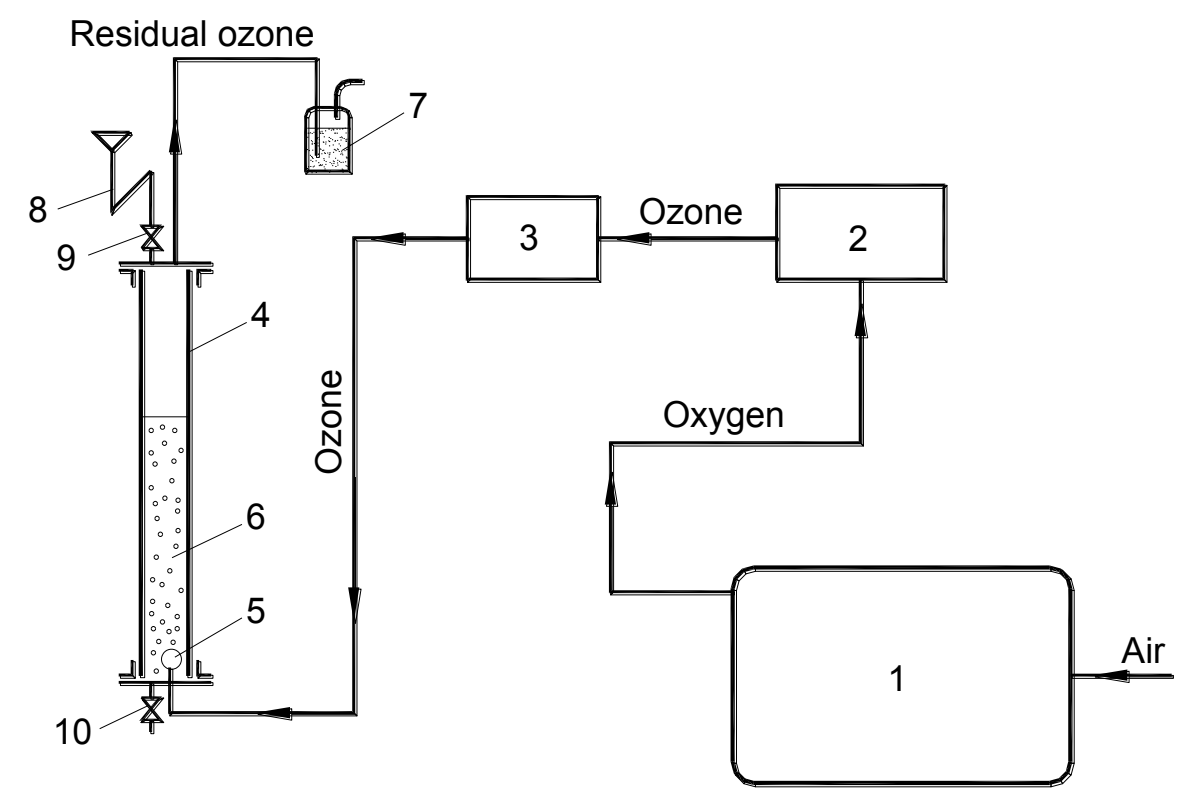

Figure 2. - Scheme of the device for obtaining an ozone solution in distilled water. 1 - oxygen concentrator Atmung OXY 6000; 2 -ozonizer PLATON 10/2; 3 - ozone meter MEDOZON 254/5; 4 - contact column; 5 - dispersant of the ozone-oxygen mixture; 6 - distilled water with dissolved ozone; 7 - destructor of residual ozone; 8 - hydraulic valve; 9 - tap for pouring distilled water; 10 - tap for sampling ozonized distilled water.

Oxygen (oxygen concentration 95\%) produced by oxygen concentrator 1 enters ozonizer 2 where ozone is produced. Ozone-oxygen mixture is fed into the contact column 4 through a porous disperser 5 while distilled water 6 is saturated with ozone. Further, the ozone-oxygen mixture depleted in ozone enters the destructor 7 which is a Drexel flask filled with a packing that degrades ozone. Ozonized distilled water 6 from the contact column 4 is taken using the valve 10 . New portions of distilled water are introduced into the contact column 4 through the hydraulic valve 8 using the valve 9.

1.5 liter flask with a ground glass stopper was used as a reactor for the interaction of ozone with an aqueous solution of the treated substance. The experiment was carried out as follows. A working solution with a volume of $500 \mathrm{ml}$ was poured into a $1.5 \mathrm{~L}$ reactor flask. To carry out the experiment, ozonized water with a volume of about 1 liter was taken from the column into a special beaker then the concentration of ozone dissolved in distilled water was determined using the MEDOZON 245/8 (Zh-30) ozonometer. For this purpose, ozonized water was taken from the middle of the beaker using a pipette and poured into a quartz cuvette of an ozone meter. The average value of four determinations was taken as the ozone concentration in the ozonized water introduced into the reaction. Next, the volume of ozonized water was calculated which must be poured into the processed sample in order to provide a given dose of ozone.After that, the calculated volume of ozonized water was measured with a measuring cylinder and added to the treated dye solution in the 
reactor flask. If the volume of the solution mixture in the coble-reactor thus obtained was less than $1 L$ then distilled water was added to the reactor flask in order to bring the volume of the mixture in it to exactly $1 \mathrm{~L}$. Thus, it was achieved that when ozone was introduced in the form of an aqueous solution, a twofold dilution of the treated dye solution with distilled water always occurred.Taking into account the above, the initial color intensity in terms of the degree of dilution of the test solutions is two times lower than that of working solutions because at a zero dose of ozone $500 \mathrm{ml}$ of distilled water must be added to $500 \mathrm{ml}$ of the working solution in order to obtain a mixture volume of 1 liter. Therefore, the initial concentration of the test solution "LanasynBlack M-DL" by the degree of dilution was 1 : 100 and for the solution "Methylene blue" - 1: 200 (at zero dose of ozone). Then, the reactor flask was sealed with a ground glass stopper and vigorously shaken for 20 minutes. The hermetically sealed reactor flask provided almost $100 \%$ use of the ozone introduced into it for the destruction of the dye under study which was in the reactor flask. Further, using the technique described in article [24], the color intensity was determined from the degree of dilution of the solution obtained as a result of ozonation.

The effect of reducing the color intensity $Э_{\text {сниж._oк }}$ is determined from the ratio:

$$
\ni_{\text {снизж._ок }}=\frac{K_{u c x}-K_{\text {обр }}}{K_{u c x}} \cdot 100 \%
$$

$K_{u c x}$ - multiplicity of dilution of the original dye solution, corresponding to the color intensity according to the degree of dilution of the original dye;

$K_{\text {обр }}$ - the multiplicity of dilution of the dye solution treated with ozone, corresponding to the color intensity according to the dilution rate.

The multiplicity of dilution is the number of volumes of distilled water that must be added to the test sample until the color completely disappears in a layer $20 \mathrm{~cm}$ high. The "Coloring-monitoring" program described in article [24] when analyzing the absorption spectrum of the test solution gives exactly the dilution factor on the basis of which the intensity of the color is determined by the degree of dilution. The multiplicity of dilution is the reciprocal of the color intensity in terms of the dilution degree, i.e. if the multiplicity of dilution is 100 then the color intensity according to the dilution degree is $1: 100$.

In Figure 3, the effectiveness of ozone is expressed through the effect of color

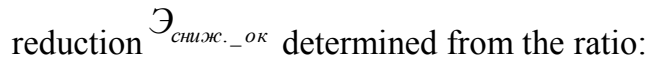

$$
\ni_{\text {сниж._ _ок }}=\frac{O_{u c x}-O_{\text {об̆p }}}{O_{u c x}} \cdot 100 \%
$$

$O_{u c x}$ - the number of volumes of distilled water that must be added to the sample of the initial dye solution until the color completely disappears in a layer $20 \mathrm{~cm}$ high.

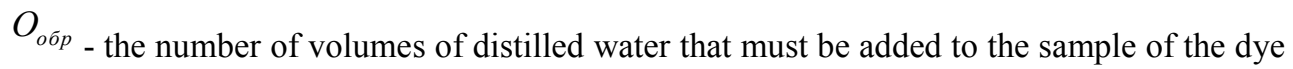
solution treated with ozone until the color disappears completely in a layer $20 \mathrm{~cm}$ high.

Spectrophotometric studies were carried out on an SF-2000 spectrophotometerto study the process of destruction of the dye under the influence of ozone. We used quartz cuvettes K10 with an optical path length of $10 \mathrm{~mm}$. The spectra of solutions of dyes in distilled water were studied before and after treatment with various doses of ozone in the visible range $(390 \ldots 760 \mathrm{~nm})$. The scanning step was $0.1 \mathrm{~nm}$ when the spectra were obtained, the number of accumulation cycles was 3. The degree of dye destruction was assessedaccording to the results of spectrophotometric studies. 


\section{Results and discussion}

Figure 3 shows the absorption spectra of the initial and ozone-treated solution of the dye "Lanasyn Black M-DL" which were recorded during the research. The change in spectra reflects the depth of destruction of the dye. Spectrum 1, corresponding to an untreated dye solution, clearly shows an absorption maximum at a wavelength of $575 \mathrm{~nm}$; however, even at an ozone dose of $0.04 \mathrm{mg} / \mathrm{mg}$ of the dye, the height of the maximum peak of the dye spectrum is compared with the height of an additional peak in the region of $400 \mathrm{~nm}$ which indicates chemical changes in the dye solution. With an increase in the specific dose of ozone, the changes in the spectra in comparison with the initial one increase, the height of the peak at a wavelength of $575 \mathrm{~nm}$ decreases and with an ozone dose of more than $0.4 \mathrm{mg}$ / $m g$ of the dye, the absorption maximum shifts to a shorter wavelength region $(400 \mathrm{~nm})$ which may indicate complete destruction of the original dye.

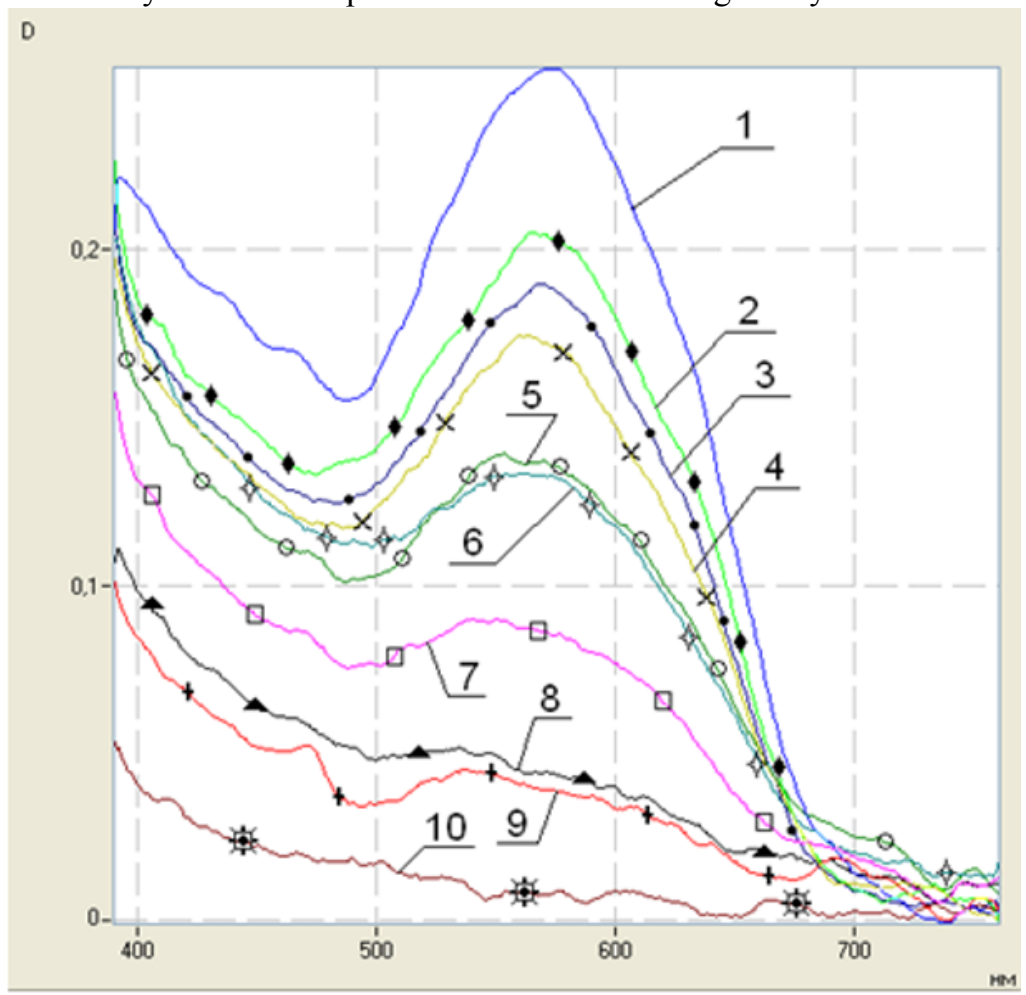

1 - initial solution;

2 - ozone dose $0.04 \mathrm{mg} / \mathrm{mg}$; 3 - ozone dose $0.06 \mathrm{mg} / \mathrm{mg}$; 4 - ozone dose $0.1 \mathrm{mg} / \mathrm{mg}$ 5 - ozone dose $0.17 \mathrm{mg} / \mathrm{mg}$; 6 - ozone dose $0.22 \mathrm{mg} / \mathrm{mg}$; 7 - ozone dose $0.27 \mathrm{mg} / \mathrm{mg}$; 8 - ozone dose $0.33 \mathrm{mg} / \mathrm{mg}$; 9 - ozone dose $0.4 \mathrm{mg} / \mathrm{mg}$; 10 - ozone dose $0.52 \mathrm{mg} / \mathrm{mg}$

Wavelength

Figure 3 - Absorption spectra of the "Lanasyn Black M-DL" dye solution treated with different doses of ozone.

Based on the obtained spectra using the above-described method, the dependence of the color intensity of the "Lanasyn Black M-DL" dye solution on the specific dose of ozone was built and it is shown in Figure 4. Analysis of this dependence shows that ozone destroys the dye at any specific dose. At the same time, to reduce the color by $81 \%$ which is equivalent to a decrease in the color intensity in the degree of dilution from 1:100 to 1:20 a dose of $0.23 \mathrm{mg}$ ozone per $1 \mathrm{mg}$ of the dye was sufficient. 


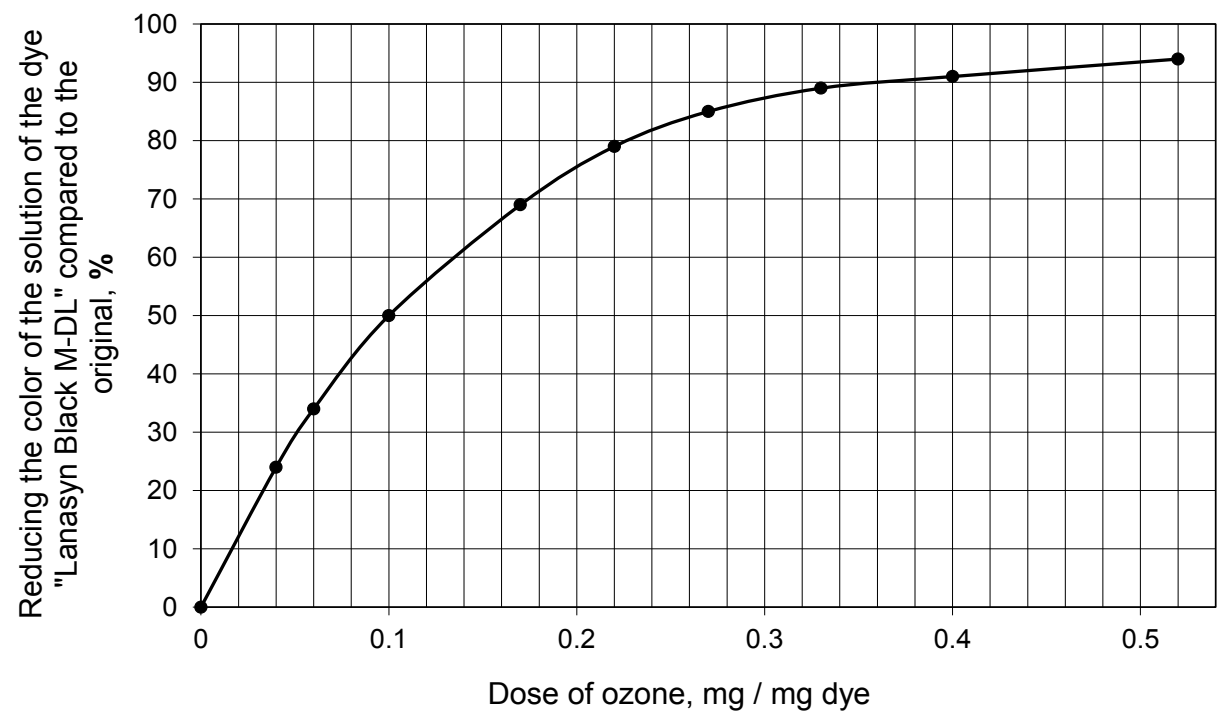

Figure 4. - The effect of the specific dose of ozone on the decrease in the color intensity of the "Lanasyn Black M-DL" dye solution.

We also investigated the effectiveness of using ozone to reduce the color intensity of the Methylene Blue dye solution. This dye does not belong to the class of azo dyes but according to the structure of the chromophore is a thiazine acid dye with weak oxidizing properties. The dye "Methylene blue" according to the literature data is relatively resistant to oxidants but it is relatively easy to recover and has a relatively low molecular weight [5] [6], therefore, according to the efficiency of its destruction using ozone and it is possible to more accurately estimate the specific doses of ozone required for the destruction of various classes of dyes.

By analogy with the previous dye, during the research, the spectra of the initial and treated with various doses of ozone solutions of the dye "Methylene blue" were recorded and it is shown in Figure 5. Changes in the spectra of the dye solution treated with different doses of ozone show a proportional decrease in absorption levels with increasing ozone dose, however, the forms the spectrum remain practically unchanged. This observation can be explained by the fact that the decomposition products of the dye absorb exclusively in the ultraviolet region, therefore, ozone also acts selectively on the molecules of this dye, i.e. on certain connections. However, the mechanism of action of ozone on dye chromophores is practically not studied. It can also be noted that, at ozone dose of more than $1.25 \mathrm{mg} / \mathrm{mg}$ of the dye spectral analysis in the visible region did not reveal noticeable absorption. 


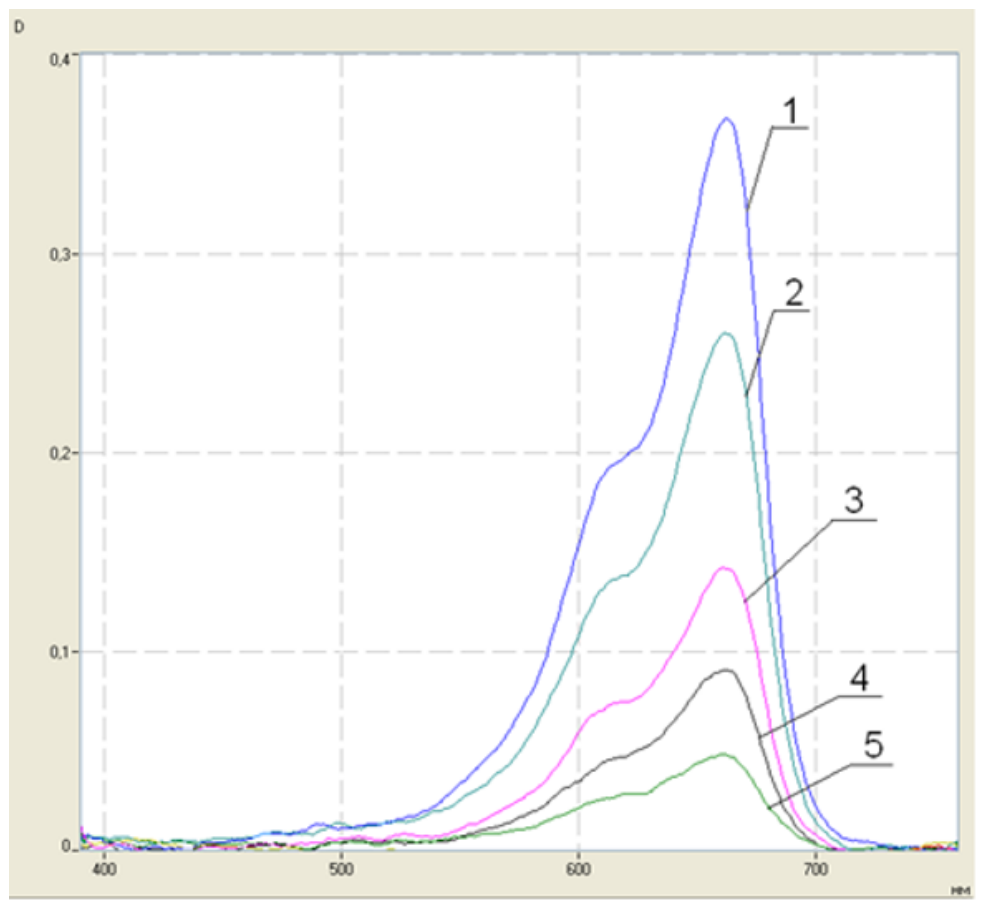

1 - initial

solution;

2 - ozone dose

$0.25 \mathrm{mg} / \mathrm{mg}$

3 - ozone dose

$0.52 \mathrm{mg} / \mathrm{mg}$;

4 - ozone dose

$0.75 \mathrm{mg} / \mathrm{mg}$

5 - ozone dose 1

$\mathrm{mg} / \mathrm{mg}$;

\section{Wavelength}

Figure 5 - Absorption spectra of the Methylene Blue dye solution treated with various doses of ozone.

On the basis of the obtained spectra, the dependence of the efficiency of ozone application for reducing the color intensity of the solution of the dye "Methylene blue" with a concentration of $2 \mathrm{mg} / \mathrm{l}$ was plotted which corresponds to the color intensity at a dilution ratio of 1: 200 and is shown in Figure 6.

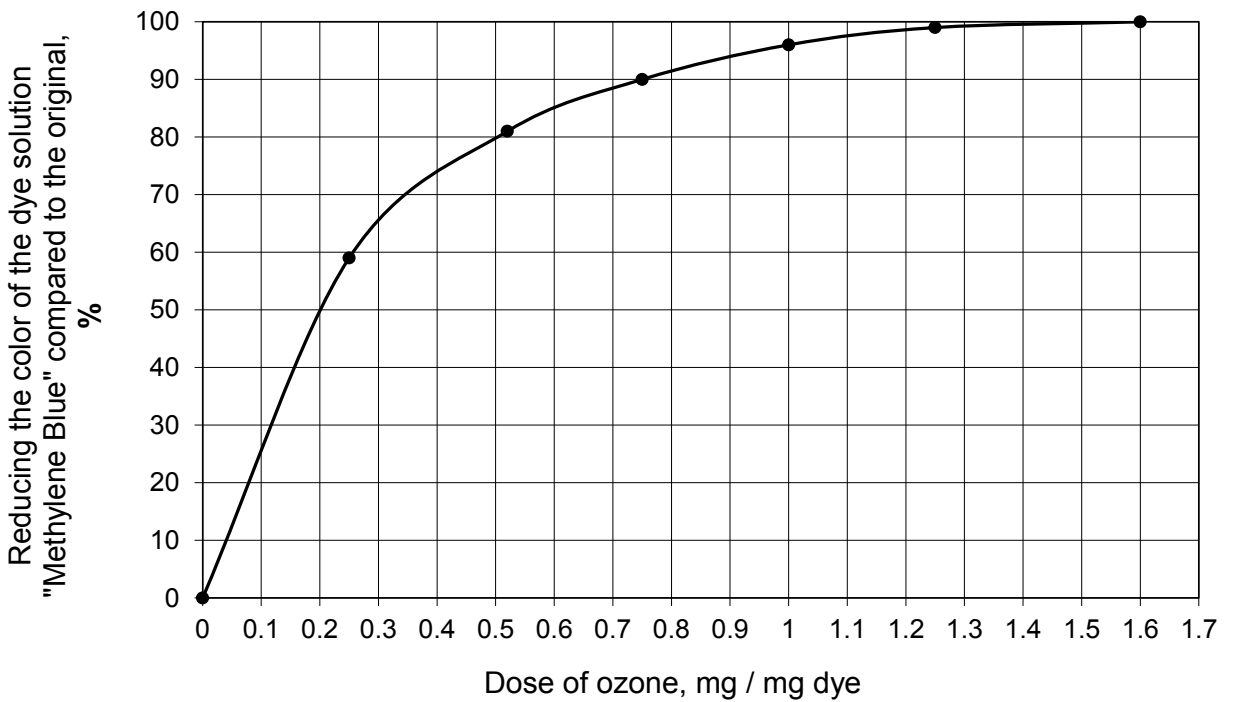

Figure 6. - Influence of the specific dose of ozone on the decrease in the color intensity of the

"Methylene blue" dye solution. 
Ozone also effectively reduces the color of the solution of this dye despite the fact that "Methylene Blue" dye is more resistant to oxidation. Thus, at a specific ozone dose of 0.75 $\mathrm{mg} / \mathrm{mg}$ of the dye, the color intensity of the ozonated model solution was already achieved at a dilution ratio of 1:20 which meets the requirements for the permissible color of wastewater discharged into the municipal drainage network. At an ozone dose of more than $1.5 \mathrm{mg} / \mathrm{mg}$ of the dye the decrease in the color intensity of the "Methylene Blue" dye solution was $100 \%$; i.e. solution became completely colorless.

\section{Conclusion}

According to the results of the studies carried out to reduce the color of solutions of dyes "Lanasyn Black M-DL" and "Methylene blue" by treatment with ozone, the following conclusions can be drawn:

1. Ozone is an effective reagent for reducing the color intensity of wastewater caused by synthetic dyes. In the studies performed, ozone acted equally effectively on dyes with different chromoform systems (on azo dye and on a dye with a heterocyclic thiazine chromophore).

2. Doses of ozone required to reduce the color intensity by $5 \ldots 10$ times according to the degree of dilution of dye solutions are $0.25 \ldots 0.75 \mathrm{mg} / \mathrm{mg}$ of the dye which in model solutions in terms of the concentration of dyes was $1.5 \ldots 3,75 \mathrm{mg}$ ozone per liter of solution. An even stronger decrease in color occurred at higher doses of ozone, up to complete discoloration.

3. The effect of color reduction under the influence of ozone is irreversible which indicates the destruction of the chromophore part of the dye molecule.

4. In view of the high reactivity of ozone which makes it possible to effectively destroy carbocyclic and heterocyclic compounds, it is possible not only to effectively reduce the color of wastewater but also to destroy the decay products of dyes which is especially important for the protection of the surrounding aquatic environment.

\section{Bibliography}

1. Vasiliev G.V., LaskovYu.M., Vasilyeva E.G. Water management and waste water treatment of textile industry enterprises. - M .: Light industry, 1976 .-- 224 p.

2. Wang Z. and others. Textile Dyeing Wastewater Treatment // Advances in Treating Textile Effluent. , 2011.

3. Yaseen D. A., Scholz M. Textile dye wastewater characteristics and constituents of synthetic effluents: a critical review // Int. J. Environ. Sci. Technol. 2019.

4. Krasnoborodko, I.G. Destructive purification of waste water from dyes / I.G. Krasnoborodko. - L: Chemistry, 1988 .-- 192 p.

5. Holkar C. R. et al. A critical review on textile wastewater treatments: Possible approaches // J. Environ. Manage. 2016.

6. Chichibabin, A.E. Basic principles of organic chemistry / A.E. Chichibabin, M: Goskhimizdat, 1958 .-- 767 p.

7. Krichevsky, G.E., Chemical technology of textile materials / G.E. Krichevsky, M.V. Korchagin, A.V. Senakhov. - M: "Light industry and consumer services", 1985. - 640 p.

8. Henze, M. Wastewater Treatment: Per. from English. / M. Henze, P. Armoes, J. La-Cour-Jansen, E. Arvan - M .: Mir, 2004 .-- 480 p. 
9. Alekseev E.V. Ecological aspects of wastewater treatment containing biologically stable organic substances // Water and ecology: problems and solutions. 2015. No. 4. P. 68-78.

10. Soares O. S. G. P. et al. Ozonation of textile effluents and dye solutions under continuous operation: Influence of operating parameters // J. Hazard. Mater. 2006.

11. Tehrani-Bagha A. R., Mahmoodi N. M., Menger F. M. Degradation of a persistent organic dye from colored textile wastewater by ozonation // Desalination. 2010.

12. Wu J., Doan H., Upreti S. Decolorization of aqueous textile reactive dye by ozone // Chem. Eng. J. 2008.

13. Turhan K., Turgut Z. Decolorization of direct dye in textile wastewater by ozonization in a semi-batch bubble column reactor // Desalination. 2009.

14. Kozhinov, V.F. Ozonation of water / V.F. Kozhinov, I.V. Kozhinov. - M .: Stroyizdat, 1974 .-- 159 p.

15. Orlov, V.A. Ozonation of water / V.A. Orlov. - M .: Stroyizdat, 1984 .-- 88 p.

16. Selcuk H. Decolorization and detoxification of textile wastewater by ozonation and coagulation processes // Dye. Pigment. 2005.

17. Snider E. H., Porter J. J. Ozone destruction of selected dyes in wastewater // Am. Dyest. Report. 1974.

18. Razumovsky, S.D. Ozone and its reactions with organic compounds / S.D. Razumovsky, G.E. Zaikov. - Moscow: Nauka, 1974 .-- 324 p.

19. Rawat D., Mishra V., Sharma R. S. Detoxification of azo dyes in the context of environmental processes // Chemosphere. 2016.

20. Neylond, O. Ya. Organic chemistry / O. Ya. Nylon. - M: Higher school, 1990 .-- 751 p.

21. Babu B. R. et al. Cotton textile processing: Waste generation and effluent treatment // J. Cotton Sci. 2007.

22. Brief chemical encyclopedia: in 5 volumes / IL. Knunyants [and others]; under total. ed. I.L. Knunyants. - M: "Soviet Encyclopedia", 1964. -T. 3.- 1112 p.

23. Turhan K. et al., Decolorization of textile basic dye in aqueous solution by ozone, Dye. Pigment. 2012.

24. Belov S.G., Naumchik G.O. Development of an instrumental method for determining the color intensity of waste water from textile enterprises // Water supply and sanitary engineering. 2017. No. 3. P. 53-65. 